\title{
Community-level density dependence: an example from a shallow coral assemblage
}

\author{
Jason E. Tanner, ${ }^{1,4}$ Terence P. Hughes, ${ }^{2}$ and Joseph H. Connell ${ }^{3}$ \\ ${ }^{1}$ South Australian Research and Development Institute (SARDI), Aquatic Sciences, P.O. Box 120, \\ Henley Beach, South Australia 5022 Australia \\ ${ }^{2}$ Australian Research Council (ARC) Center for Excellence in Coral Reef Studies, James Cook University, \\ Townsville, Queensland 4811 Australia \\ ${ }^{3}$ Department of Ecology, Evolution and Marine Biology, University of California, Santa Barbara, California 93106 USA
}

\begin{abstract}
While density dependence is a popular topic of research in population ecology, it has received much less attention at the community level. Using 27 years of data from Heron Island, on Australia's Great Barrier Reef, we develop a matrix model of coral community dynamics that shows that community-level density dependence does occur and that it is fairly common, being found in $38 \%$ of the model parameters for which it was tested. In particular, colonization of free space (through either recruitment or growth of existing colonies) was nearly always density dependent. There were no consistent patterns in the results for mortality, persistence, or species interactions. Most transitions were found to be dependent on the cover of the incoming species group, with only a few dependent on that of the outgoing species group. In addition, few of the transitions representing species interactions were dependent on the amount of free space present, suggesting that the cover of other species does not influence encounters.

When these results were combined into a model of community dynamics, it was found that density dependence resulted in a moderate increase in coral cover, which was spread over most species groups. The dynamics of the density-dependent assemblage were also a lot noisier than those of an assemblage without density dependence. Sensitivity analysis indicated that it was density dependence in the colonization probabilities, particularly of encrusting acroporids, bushy Acropora and staghorn Acropora, which had the main influence on the model, although persistence of free space was also important. Transitions representing mortality were only of minor importance, and those representing species interactions were of no importance.
\end{abstract}

Key words: colonization; community dynamics; competition; coral reefs; density dependence; disturbance; Great Barrier Reef; matrix model.

\section{INTRODUCTION}

The effects of density on individual organisms and on population dynamics have received wide attention in many different ecological systems (e.g., plants, Peters 2003; vertebrates, Wang et al. 2006; insects, Miller 2007; marine invertebrates, Tanner 1997, 1999). However, the importance of density dependence in determining population dynamics is still widely debated (e.g., Peters 2003, Brook and Bradshaw 2006). Possibly as a result of this debate, the consequences of density dependence at the community level have been largely ignored. Different ecological processes may come into play when a population is at high density compared to low (Walker and Chapin 1987), resulting in substantial changes to the way the community in which it is contained functions. For instance, a species capable of inhibition (sensu Connell and Slatyer 1977) is unlikely to have much effect

Manuscript received 25 February 2008; accepted 8 May 2008; final version received 26 June 2008. Corresponding Editor: M. A. Hixon.

${ }^{4}$ E-mail: Tanner.Jason@saugov.sa.gov.au at low densities, but at high densities can prevent other species from colonizing (e.g., Prach et al. 1996).

An example of the importance of density in determining community dynamics may be seen in the situation in which alternative stable states exist. In these situations, the community persists for a long term in one of two or more states, but occasionally flips between states, often in response to a disturbance (e.g., Beisner et al. 2003, Petraitis and Dudgeon 2004). As an example, Italian rock pools may be dominated either by canopyforming macroalgae or turf-forming algae, depending on the timing of disturbance in relation to recruitment of the macroalgae (Benedetti-Cecchi 2000). If free space becomes available when the macroalgae are recruiting, then macroalgae are able to establish and suppress the turf algae; otherwise the reverse occurs. For both groups, colonization success is dependent on the density of the other, and this has important implications for the way the assemblage develops. Indeed, even if alternate stable states are not possible for a given community, disturbance may still alter competitive rankings (Suding and Goldberg 2001), resulting in the potential for density dependence to operate at the community level. 
Several recent theoretical studies have highlighted the likely importance of density dependence for determining community dynamics (e.g., Molofsky and Bever 2002, Eppstein et al. 2006), and it is likely to be important in explaining discrepancies between the outputs of simple transition models and reality (Greene and Schoener 1982). The emerging consensus is that the nature of frequency dependence has a strong influence on species coexistence and diversity and that its presence can explain the ability of invasive species to become established. Feagin et al. (2005) showed that facilitation in a dune plant assemblage, or positive density dependence at the community level, was required to accurately mimic observed dynamics. Few other modeling studies have expressly examined the role of density dependence in determining community dynamics and assessed which parameters actually determine the manner in which the community develops.

In this paper we aim to determine whether community-level density dependence occurs in a well-studied shallow reef crest coral assemblage. We then go on to assess what role such density dependence might play in determining the dynamics of the assemblage. For the latter, we extend our existing models of dynamics in these assemblages (Tanner et al. 1994, 1996) to include density dependence in the parameters in which it was detected. This is necessary, as knowledge of the existence of density dependence does not tell us whether it is biologically relevant (e.g., Hill et al. 2002) or how it affects the community. While for many organisms, density is expressed as the number of individuals per unit area, for clonal organisms that have large variation in size and for which competition is often size dependent, percent cover provides a more biologically relevant measure.

In sessile communities such as those studied here, the rate of colonization of free space (whether by recruitment or by growth of existing colonies) may increase with density, at least initially. This increase would primarily be due to an increase in the number of individuals present that can grow into free space, although there may also be a positive effect of early colonizers on later colonizers (i.e., density-dependent recruitment). As density increases further, however, and colonies start to compete with one another, colonization of free space may begin to decline (e.g., Tanner 1997). Competition between species may also be affected by density, especially if processes such as facilitation and inhibition (Connell and Slatyer 1977) are operating. Caswell and Cohen (1995) model this in a metapopulation context. Thus in the case of a forest, fast-growing, shade-intolerant species can dominate slower growing, shade-tolerant species early in succession when overall density is low, but the shade-tolerant species dominate later when density is high (e.g., Hartshorn 1978). In coral communities, fast-growing tabular Acropora can dominate over slow-growing massive species early in succession when cover is low and most colonies are small, but the massives can dominate later when cover is higher and colony size increases. Aggressive sponges are also more abundant on Caribbean coral reefs at high coral cover, making the rate of competitive encounters and overgrowths between these two groups a function of coral density (Aerts 1998). Using the same data as used here, Spencer and Tanner (2008) show that a continuous time density-dependent model gives a better fit to observed dynamics than do several non-density-dependent models. However, they do not examine in detail density dependence in individual parameters or its consequences for community dynamics.

\section{The Model}

The model used here is an elaboration of a Markov matrix model originally used by Horn (1975) to model succession in temperate forests of North America. Markov models track the state of a series of ecological units (in this case points in space) through time, based on the assumption that the state at any given time $t+1$ is dependent solely upon its state at time $t$. The model can be represented as a square matrix of transition probabilities that describes the probability that a point in space occupied by species $x$ at time $t$ will be occupied by species $y$ at time $t+1$. This matrix includes four different types of transitions. (1) There are self-self transitions along the diagonal. These generally represent persistence, although a small proportion will represent instances of colony death followed some time later by colonization of the same taxon. (2) There are transitions to free space, which is included in the model as an ecological state. These transitions represent a measure of disturbance, which may be due to either large-scale rare events such as cyclones or small-scale routine events such as wave action and predation. In the model represented here, these transitions are in the bottom row of the matrix. (3) Colonization probabilities are transitions from free space to a species and, in this case, are in the last column of the matrix. Colonization may be either via recruitment of new individuals or growth of existing individuals. (4) The remaining elements of the matrix represent the probabilities of transition from one species group to another. As with persistence, while most of these transitions will be direct, some will be via a third state (see Spencer and Susko 2005).

This model is analogous to the stage-classified Leslie matrix model described and reviewed in detail by Caswell (2001) and can be analyzed using the same techniques. So far, the model has been used primarily in its linear form, with constant transition probabilities, to model succession in forests (Horn 1975, Enright and Ogden 1979), alpine assemblages (Lough et al. 1987), termite communities (Usher and Parr 1977), temperate marine assemblages (Hill et al. 2002, Wootton 2004), and coral reefs (Tanner et al. 1994, 1996). While relatively simple, in at least one system this model has performed surprisingly well at predicting the consequences of experimental manipulations (Wootton 2004). 
We know of only two instances of the model being extended to incorporate nonlinear transition probabilities. Acevedo (1981) introduced a model of forest dynamics in which the transition probabilities were based on the densities of the different species. However, he concentrated on examining simple two-species communities analytically, rather than a full densitydependent model in detail. Caswell and Cohen (1995) also used a simple nonlinear model to examine the consequences of facilitation and inhibition in a theoretical community. Here we extend Acevedo's (1981) approach and develop a community model of a shallow reef crest coral assemblage in which the transition probabilities are functions of the cover of the different species groups in the model. In a companion paper (Spencer and Tanner 2008), we also examine a densitydependent continuous time version of the model.

Community development through time in this model can be simulated using the following equation:

$$
\mathbf{x}(t+1)=\mathbf{A}[\mathbf{x}(t)] \mathbf{x}(t)
$$

where $\mathbf{x}(t)$ is a column vector that describes the community composition (i.e., percent cover of each species group) at time $t$, and $\mathbf{A}[\mathbf{x}(t)]$ is the transition probability matrix that has entries that are a function of current community composition. Due to the complex nonlinear nature of this model, it is not amenable to the analytical techniques usually employed with matrix models, but must instead be examined primarily by simulations.

In most of the assemblages studied using this model so far, it has proven to be ergodic in both its linear and nonlinear forms. Ergodicity is the property whereby the model forgets its past, and no matter what the starting point, the same equilibrium composition is always reached (see Caswell 2001). This suggests that these models lack sufficient complexity to account for multiple stable states. Given the more complex nonlinear nature of the model presented here, however, this may not be the situation. Indeed, the only exception to the general finding of ergodicity was in the more complex model of Acevedo (1981), in which the equilibrium composition was highly dependent on the initial community composition.

\section{Methods}

To examine how density and community composition affect the rates of transition between different species within shallow reef crest coral assemblages, we used density-dependent transition matrix models. These models were parameterized with data from a long-term monitoring study of the coral reef at Heron Island, at the southern end of Australia's Great Barrier Reef $\left(23^{\circ} 26^{\prime} \mathrm{S}, 151^{\circ} 55^{\prime} \mathrm{E}\right)$. This study covers three distinct sites, and while models were examined for each site, only the protected (south) crest is presented in detail here for brevity (see Plate 1). The choice of site was made for consistency with Tanner et al. (1994). At the protected (south) crest site, a total of 17 censuses of three permanently marked $1-\mathrm{m}^{2}$ quadrats were conducted, with a census interval of $19 \pm 2$ months (mean \pm SE). At the exposed (north) crest, four quadrats were censused 12 times and one 10 times between 1962 and 1989, with a census interval of $26 \pm 5$ months, and at the exposed (north) pools, two quadrats were censused 14 times, with an interval of $26 \pm 4$ months. The two exposed sites are vulnerable to cyclones, whereas the protected site is usually sheltered by neighboring Wistari Reef (Connell et al. 1997). At each census, each quadrat was photographed from directly overhead, with the photographs subsequently being traced onto half life-sized maps. To calculate the rates of transition between each species for each quadrat and census interval, a $20 \times 20$ regular square grid of points was overlaid on each map, and the species present under each point were recorded and compared for successive censuses (19200 transitions were scored in total at the protected crest, 23200 at the exposed crest, and 11200 at the exposed pools). The steel stakes permanently marking each corner of the quadrats ensured that movement of the points was minimized, and only became an issue when cyclones removed one or more stakes. On such occasions, the photographs and maps were carefully compared to identify other reference points, and the vast majority of transitions were to free space anyway.

As the benthic assemblage was highly complex, with 72 coral and nine algal species being recorded in the quadrats (Connell et al. 1997), species were grouped to enable transition rates to be summarized efficiently and to ensure adequate sample sizes for analysis. Each of the species groups (Table 1) was based on both taxonomic and morphological criteria, ensuring that their members were as similar as possible. Six scleractinian coral groups were chosen, along with one each for soft corals and algae. We also employed free space as a ninth group to account for all possible states. Free space lacks corals and macroalgae, but is usually occupied by crustose coralline or turfing algae, and is potentially available for corals to recruit onto or grow over. The use of nine species groups results in 81 possible transitions between groups, including transitions to self. More detailed descriptions of the site and methods can be found in Tanner et al. (1994, 1996) and Connell et al. (1997, 2004).

The effects of density and community composition on the transition probabilities were examined separately for each of the 81 transitions at each site. For each transition, the transition probability was regressed against the cover of each of the species groups involved and that of free space. Each census and each quadrat were regarded as separate points for this analysis, meaning that each regression is potentially based on 51 data points at the protected crest, 53 at the exposed crest, and 26 at the exposed pools. The cover of each species group was obtained by expressing the number of points scored for that group as a proportion of the total 
TABLE 1. Species transition matrix for the protected crest showing those transitions that were found to be density independent (values from Tanner et al. [1994: Table 2]) or density dependent (indicated by "d").

\begin{tabular}{cccccccccc}
\hline \hline Species & $\mathrm{A}$ & $\mathrm{B}$ & $\mathrm{C}$ & $\mathrm{D}$ & $\mathrm{E}$ & $\mathrm{F}$ & $\mathrm{G}$ & $\mathrm{H}$ & $\mathrm{I}$ \\
\hline $\mathrm{A}$ & 0.354 & $\ldots$ & $\mathrm{d}$ & $\mathrm{d}$ & 0 & 0.071 & $\mathrm{~d}$ & $\ldots$ & $\mathrm{d}$ \\
$\mathrm{B}$ & $\mathrm{d}$ & 0.314 & 0.005 & 0.004 & $\cdots$ & $\cdots$ & 0 & 0 & $\mathrm{~d}$ \\
$\mathrm{C}$ & $\mathrm{d}$ & 0.03 & $\mathrm{~d}$ & $\mathrm{~d}$ & $\cdots$ & $\mathrm{d}$ & $\mathrm{d}$ & $\cdots$ & $\mathrm{d}$ \\
$\mathrm{D}$ & $\mathrm{d}$ & 0.016 & $\mathrm{~d}$ & 0.439 & $\cdots$ & 0.057 & 0.031 & $\cdots$ & $\mathrm{d}$ \\
$\mathrm{E}$ & $\cdots$ & $\cdots$ & $\cdots$ & $\cdots$ & $\cdots$ & $\cdots$ & $\cdots$ & 0 & $\mathrm{~d}$ \\
$\mathrm{~F}$ & 0.009 & $\cdots$ & $\mathrm{d}$ & $\cdots$ & 0 & $\cdots$ & $\mathrm{d}$ & 0 & 0.007 \\
$\mathrm{G}$ & 0.015 & $\cdots$ & 0.013 & 0.014 & $\cdots$ & 0.052 & 0.34 & 0 & $\mathrm{~d}$ \\
$\mathrm{H}$ & $\cdots$ & $\cdots$ & $\cdots$ & $\cdots$ & 0 & 0 & 0 & $\cdots$ & $\mathrm{d}$ \\
$\mathrm{I}$ & $\mathrm{d}$ & $\cdots$ & $\mathrm{d}$ & $\mathrm{d}$ & $\cdots$ & 0.678 & 0.501 & $\cdots$ & $\mathrm{d}$ \\
\hline
\end{tabular}

Notes: Ellipses indicate transitions for which there were insufficient data to test density dependence. Transitions that did not occur are indicated by 0 . Transitions are from the species group in the column headings to the species group in the left column (A, encrusting acroporid corals; B, tabular Acropora; C, bushy Acropora; D, staghorn Acropora; E, soft corals; F, algae; G, massive corals; $\mathrm{H}$, pocilloporid corals; I, free space). Models were parameterized with data from a long-term monitoring study of the coral reef at Heron Island, at the southern end of Australia's Great Barrier Reef.

number of points scored. Because there are multiple data points for each quadrat, the data for these regressions are not independent, and thus significance levels were calculated using randomization procedures (Manly 1991). Data were first randomized within quadrats and then between quadrats as a block to preserve the temporal patterns in cover. Generalized additive models (Hastie and Tibshirani 1990) were used to perform the regressions, as there was no reason to believe that the relationship between transition probabilities and cover would be linear (see Abrams 2001).

Each transition probability was modeled as a function of the cover of each species group involved and of free space using a binomial distribution (for probabilities). So for the transition $X Y$ three models were developed, one with $X Y$ as a function of the cover of $X$, one as a function of $Y$, and one as a function of free space. If more than one model was significant, they were then compared using an analysis of deviance, and the best model was selected for further study. The effects of adding a second variable were also investigated, and in no case was the third variable significant. Each regression was then checked for outliers, and these were removed if the species present at the start of the census interval was represented by less than five points on the grid. All probabilities of zero that were based on fewer than five points, including when the incumbent species was absent from the quadrat, were also removed. Outliers based on more than five points appeared to be real observations and so were retained in the data set.

While the choice of five points is somewhat arbitrary, transition probabilities based on fewer points are almost certainly heavily influenced by chance, and those based on more than five points were very rarely outliers. In addition, only those models based on transition probabilities with at least five nonzero values were retained for further consideration. When fewer than five nonzero values existed, there were problems with obtaining convergence in the model fit, so the model could not be reliably estimated.

Finally, the selected generalized additive models were compared to the equivalent generalized linear models to determine whether the nonlinear component added significantly to the model fit. If it did not, only the linear model was examined further. A significance level of $\alpha=0.05$ was used for all tests, as it was considered that applying a Bonferroni correction would prevent important density-dependent transitions from being detected (modified $\alpha=0.0004$ ). Thus, purely by chance we would expect to see aproximately seven significant results across the three sites.

To determine how density dependence affects community development, the density-dependent transition probabilities were combined into a matrix model of community dynamics. Transition probabilities for those matrix elements that were found to be density independent, or for which density dependence could not be tested, were obtained from Tanner et al. (1994: Table 1), which gives mean transition probabilities for the entire 27 -year study period. This model was then used to simulate community development over time using Eq. 1, starting with $100 \%$ free space, thus simulating succession after a major disturbance such as a cyclone. At each iteration, the transition probabilities had to be standardized. This was necessary as the total probability of species group $x$ going to another species group (including persistence) must be one, since space in the model would otherwise either shrink or grow. Standardization was achieved by summing the probabilities of transition for each species and dividing the transition probabilities by the sum for that species.

The above simulations started with $100 \%$ free space, to mimic succession after a major disturbance. It is possible, however, that different model communities (or alternate stable states) could develop depending on the initial starting conditions if the model is not ergodic. To investigate the importance of the starting community 
composition, a series of simulations was run with a range of other starting conditions, including $100 \%$ cover of each species group in turn, as well as mixtures of different groups.

While individual transition probabilities may be density dependent, this density dependence may not have any effect at the community level. The importance to community development of density dependence in each transition was examined by means of a sensitivity analysis. While analytical procedures have been developed for sensitivity analysis of density-dependent population models (Grant and Benton 2003, Caswell et al. 2004), they are not applicable here. Thus, sensitivity analysis was conducted by examining the consequences of dropping density dependence in each term individually. The effect of this procedure on both transient dynamics and equilibrium community composition was examined. For these simulations, densitydependent transition probabilities were replaced by the equivalent density-independent probability from Tanner et al. (1994: Table 1).

\section{RESULTS}

\section{Transition probabilities}

At all three sites, the density of one or more species groups was important in determining a large number of transition probabilities. Of the 81 possible transitions between species groups, only 10 did not occur at the protected crest (Table 1), seven at the exposed crest, and nine at the exposed pools (Tanner et al. 1994: Table 2). Another 28, 24, and 29 transitions occurred in less than five combinations of quadrat and census interval and were not tested for density dependence because of low sample size. Of the remaining 43 transition probabilities at the protected crest 24 showed some form of significant density dependence (at $\alpha=0.05$ ). This decreased to 14 out of 50 at the exposed crest and 13 out of 43 at the exposed pools, which are both exposed to a higher degree of wave disturbance. At the protected crest, it was more common for the density of the species group colonizing a point to be important (significant for 22 transitions) than it was for the density of the species group being displaced (significant for 10 transitions, eight of which were colonization or persistence of free space). At the exposed crest the density of the colonizing species was significant on 12 occasions and that of the incumbent species on nine occasions, with the equivalent numbers at the exposed pools being 12 and one (with the one being a self-self transition). Moreover, in nearly all cases in which the density of the incumbent was important, it was less important than that of the colonizing species. The density of free space was significant for 21 transitions at the protected crest, nine at the exposed crest, and two at the exposed pools, although 10 and eight of these were transitions involving free space for the protected and exposed crests, respectively. There are no apparent patterns in which transitions are and are not density dependent, with the exception that nearly all coloniza- tion transitions are (eight of nine for each crest site and five of nine for the pools).

For the most part, the generalized additive models showed that the relationships between the transition probabilities and the cover variables were nonlinear (Appendix A). Only the results for the protected crest are considered in detail here, as they are broadly typical of the other two sites (J. E. Tanner, unpublished data). Density-dependent transition probabilities fall into three general categories (see Table 1 for transition abbreviations). (1) There are those that decrease with the cover of a given species group. This is typified by the transition representing persistence of bushy Acropora (CC), which decreases as the amount of free space increases. (2) There are those in which the transition probability increases with cover over all or part of the range. Examples of the former are the transitions from free space to encrusting acroporids (IA), bushy Acropora (IC), and staghorn Acropora (ID). As the cover of each group increases, its ability to colonize free space also increases, presumably due to growth. Examples of the latter are the transitions from massives to encrusting acroporids (GA) and bushy to staghorn Acropora (CD), in which, at lower levels of cover of the incoming species group, its ability to acquire space increases, but this reaches a plateau at higher levels of cover. (3) There are a number of transitions that peak at intermediate levels of cover and then decline. Examples of this pattern include the transitions from staghorn Acropora and massives to bushy Acropora (DC and GC, respectively) and possibly from free space to soft corals (IE), although the relatively few transitions at high cover make the later result uncertain.

While some of these relationships explain very little of the variation in the data (e.g., 13\% for the DI transition; Appendix A), others explain a large proportion (e.g., $74 \%$ for the IE transition). Five of the models that explain $>50 \%$ of the variation in the data are for colonization (and persistence) of free space, with another two in this group explaining $>40 \%$ and only one being $<40 \%$. In contrast, only two models for interaction, disturbance, and persistence of living groups explained $>50 \%$ of the variation in the data, with another two explaining $>40 \%$ and 12 explaining $<40 \%$.

\section{Simulations}

The influence that the densities of the different species groups have on community development was investigated by simulating successional sequences from $100 \%$ free space and comparing these sequences to the sequences generated from non-density-dependent models in Tanner et al. (1994). The effects on community dynamics of accounting for density in the transition process are relatively small, although there are some distinct differences between development of a densitydependent community and a non-density-dependent community (Fig. 1). One difference between the two models is that in the density-dependent simulation, it takes considerably longer for equilibrium to develop, up 

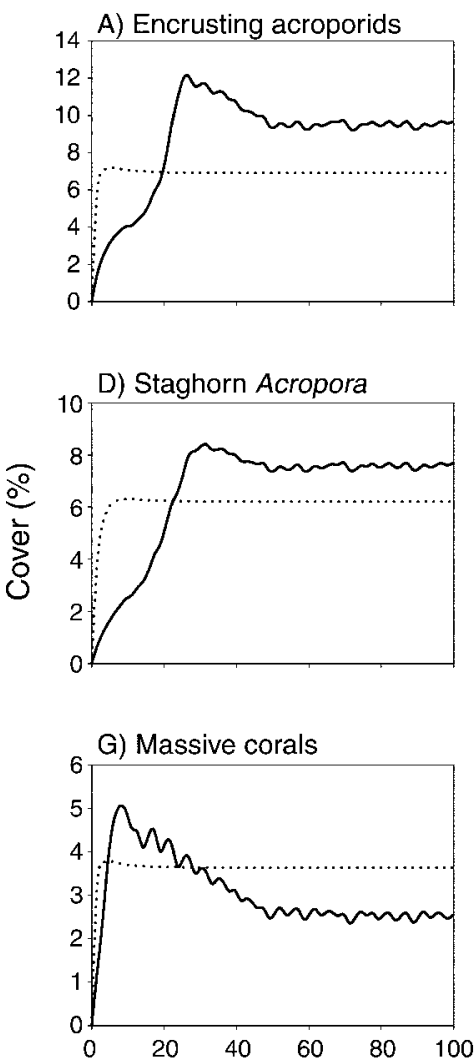
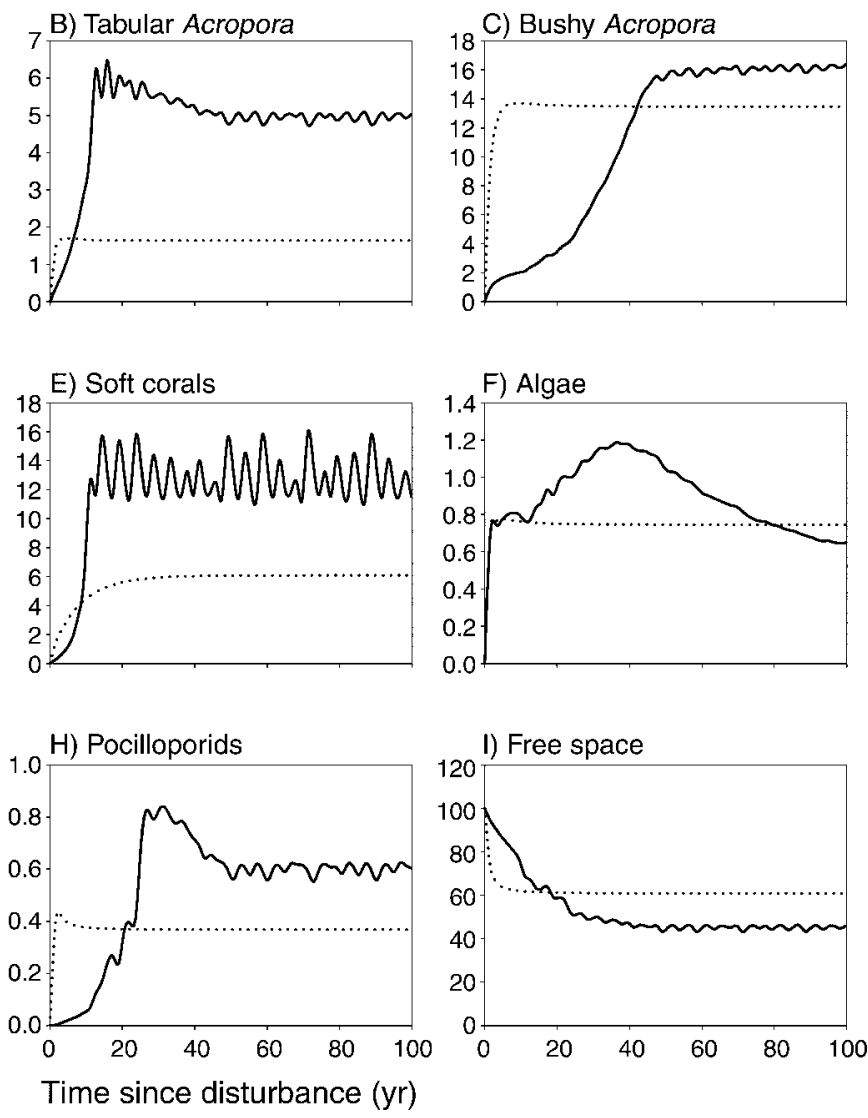

FIG. 1. Density-dependent (solid lines) and density-independent (dotted lines) simulations for the protected crest, starting from $100 \%$ free space. Note differences in scales. Models were parameterized with data from a long-term monitoring study of the coral reef at Heron Island, at the southern end of Australia's Great Barrier Reef.

to $\sim 100$ years at the protected crest, due to the very slow decline in massive corals $(\mathrm{G})$, in comparison to $\sim 20$ years in the non-density-dependent simulation. Hard coral cover also takes much longer to reach its peak in the new model, and most species groups show a more rounded peak than was observed previously. There is a tendency for overshoots of equilibrium abundance to be much larger, and to last longer, with massives (G) and algae $(F)$ reaching about twice their final abundance within 5-10 years and 30-40 years, respectively. Several species groups take a relatively long time to become established at some sites. The best example of this is bushy Acropora (C), which show an increasing rate of increase for the first 50 years before reaching equilibrium, and pocilloporids $(\mathrm{H})$, which are almost absent for 25 years and then suddenly jump in cover. The densitydependent model also produces oscillations in cover, especially for soft corals (E), which are not present in the original model. These oscillations are probably an artifact of the 19-month time step, however, which causes sudden changes in cover every 19 months.

Examination of the equilibrium abundances produced by the density-dependent model (Fig. 2) shows several important differences from the non-density-dependent model. At the protected crest, there is a substantially higher cover at equilibrium when density is included in the model ( $55 \%$ vs. $39 \%$ ), with the increase being spread over most species groups. Massive corals $(\mathrm{G})$ and algae (F) were the only groups with a lower equilibrium cover in the density-dependent model than the non-densitydependent model. The greatest increases in modeled cover were for tabular Acropora (B, 303\%) and soft corals (E, 218\%).

The model for the protected crest showed almost no dependence on starting community composition, that is, it appears to be ergodic in nature, forgetting its past. The only exceptions were cases in which soft corals (E) exceeded $29 \%$ cover, when soft coral cover at equilibrium became $71 \%$, with decreases in most other species groups (Fig. 3). It is of course possible that there are other starting communities that will result in a different equilibrium community developing, but given the wide range of initial conditions examined, this seems unlikely.

\section{Sensitivity analysis}

Sensitivity analysis of the effects of density dependence showed that density dependence in colonization has the greatest effect, along with the persistence of free 


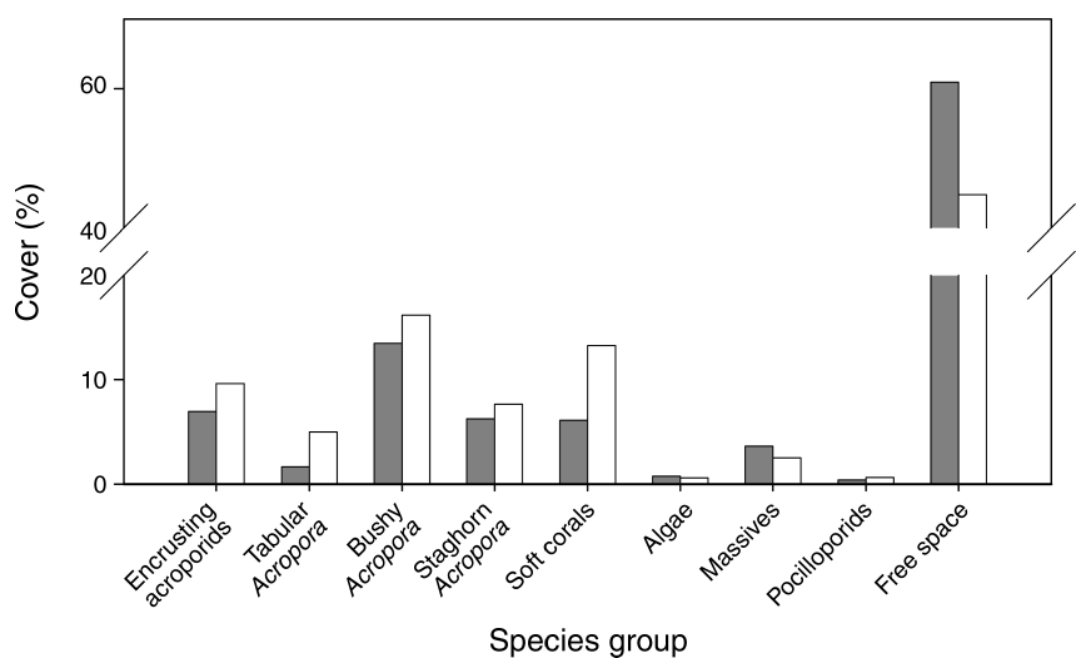

FIG. 2. Comparison of equilibrium cover for density-independent (gray) and density-dependent (white) models.

space (Appendix B). In particular, colonization by encrusting acroporids, bushy Acropora, and staghorn Acropora (the IA, IC, and ID transitions) had very high sensitivities, along with the II transition (persistence of free space). For these four transitions, soft corals (E) increased by $>400 \%$ when density dependence was eliminated in the transition, with equilibrium cover of all other species groups except massives $(\mathrm{G})$ decreasing by between $50 \%$ and $90 \%$. Eliminating density dependence in colonization of table Acropora, soft corals, and pocilloporids (the IB, IE, and IH transitions) had some effect, but much less, as did eliminating it in the disturbance of encrusting acroporids (IA). No transitions involving interactions between two different species groups were of any importance, with eliminating density dependence in these only having small effects on both equilibrium abundance and transitory dynamics.
The only important mortality probability was that of encrusting acroporids (AI).

\section{DisCUSSION}

\section{Density-dependent transitions}

Significant density dependence was found in a moderate proportion of the transition probabilities in which it could be tested (28-56\%, depending on site). The density-dependent transitions are found throughout the matrix, although nearly all colonization probabilities are density dependent, whereas mortality generally was not. This is similar to North American hardwood forests, in which ingrowth (or recruitment) and growth probabilities (between them equivalent to colonization) were generally dependent on stand basal area, but mortality rates were not (Lin et al. 1996). In contrast, in

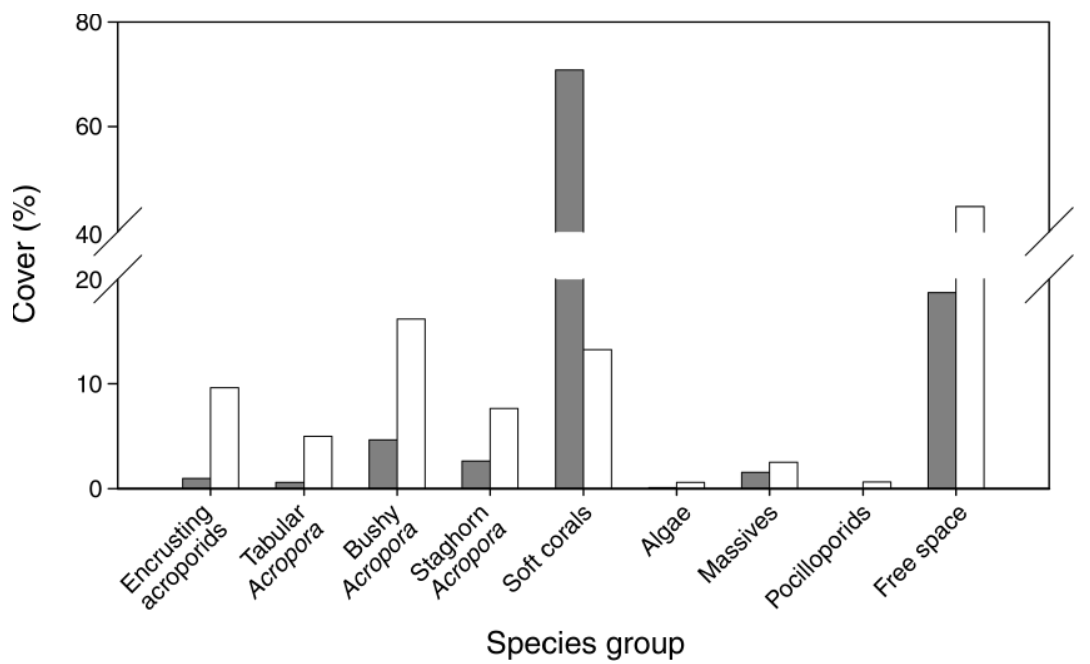

FIG. 3. Results of analysis to determine model ergodicity. Gray bars indicate equilibrium composition when soft corals (E; see Table 1) commenced with $>29 \%$ cover, and white bars indicate the equilibrium composition for all other simulations (including starting with $100 \%$ free space). 

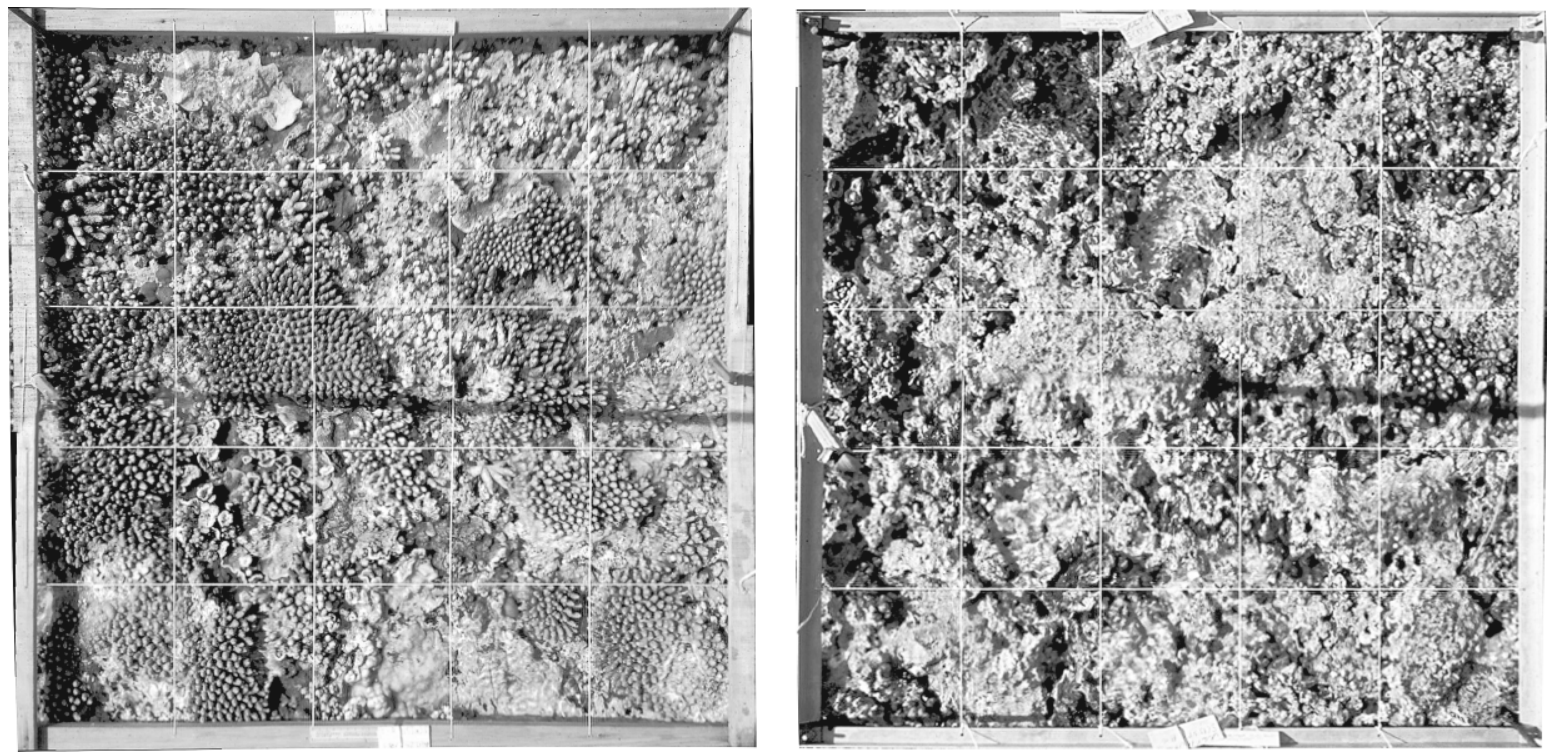

Plate 1. The protected crest B quadrat in (left) 1965 and (right) 1972, showing changes brought about by a cyclone in 1972 Photo credits: J. H. Connell.

species-rich tropical forests, Peters (2003) found extensive community-level density dependence in tree mortality. Density accounts for substantially less variation in the parameters of the forest model of Lin et al. (1996), where $R^{2}$ was generally $<0.1$, than for the coral model presented here, in which up to $74 \%$ of the variation was explained for some transitions (see Appendix A). Buongiorno et al. (1995) found similar results for a softwood forest in France and additionally showed that recruitment of each species was a positive function of adult density, which agrees with our finding that colonization is generally positively related to a species' cover.

The observation that transitions that were dependent on the cover of the incoming species are almost invariably positively related to that species' cover is consistent with other results that suggest that competition among clonal organisms is often size dependent (e.g., Brace and Pavey 1978, Buss 1980). It is likely that at high densities there is a greater proportion of large colonies, thus increasing competitive success. For the same reasons, it would perhaps be expected that a species would be more successful at holding onto space as its density increased, resulting in transitions being positively dependent on the density of the incumbent species also. This is not the case, however, with transitions being both positively and negatively dependent on the cover of the incumbent species. In part, this pattern may be due to larger colonies being more susceptible to mortality through routine disturbance.

Several studies have examined density-dependent competition in plant assemblages, and the results of these are inconsistent, with some species in some studies affected by density and others not (Taylor and Aarssen
1989, Pantastico-Caldas and Venable 1993, Zamfir and Goldberg 2000, Suding and Goldberg 2001). For example, Goldberg et al. (2001) found that the direction and extent of community-level density dependence in annual plant communities varied substantially between life-history stages, with both positive and negative effects present. Thus, competitive rankings did not remain consistent between stages, although in this case they did not vary with density within a stage. Conversely, Pantastico-Caldas and Venable (1993) found that competitive ranking between two desert annuals could vary between densities, and similar results have been found in a number of traditional replacement series experiments on plant competition (reviewed in Taylor and Aarssen 1989). Our study shows a similar lack of consistency with regard to the effects of density on transition rates between coral species, possibly due to the wide range of competition mechanisms corals employ (Lang and Chornesky 1990). Community-level density dependence still requires much further study before any generalizations about when and where it will occur can be drawn.

\section{Community-level effects of density}

Adding density dependence to models of coral reef community dynamics has an obvious effect on model community development, with higher coral cover present at equilibrium, as shown in Fig. 1 (for all three sites; J. E. Tanner, unpublished data). Thus, if density dependence were not occurring, we would expect to see fewer corals and more free space. In addition, density dependence adds apparent noise to changes in coral cover, with some groups oscillating substantially through time. 
While a semi-Markov model of this assemblage also produced substantial short-term variation in transitory dynamics, this quickly died out, and all other models of the assemblage have displayed a smooth approach to equilibrium (Tanner et al. 1996). The slow establishment of some species groups in the density-dependent model is also a major difference from the non-density-dependent model, and in the case of algae (F) and pocilloporids $(\mathrm{H})$ it is rather surprising. These two groups are generally regarded as early colonizers and poor competitors, which peak early in succession before declining (pocilloporids, Harrison and Wallace 1990; algae, Connell 1987). For pocilloporids $(H)$, this pattern can be explained by the lack of colonization events observed when there were high levels of free space (Appendix A). Obviously, at this site, the members of the group are not good colonizers. Colonization of algae (F) was density independent (Table 1), and it is unclear why it shows such a delay in establishment. The long census interval (19 months) does mean, however, that most of the early colonizing species were not recorded and therefore not included in the model. Similar patterns occur in a continuous time model of this assemblage (Spencer and Tanner 2008), with substantial oscillations in cover of all species groups. It must be stressed that the model is simply a tool to examine the potential consequences of density dependence at the community level and cannot predict community dynamics. Indeed, given the high level of stochasticity in the disturbance regime and the long-term gradual changes in environmental conditions at the site (Connell et al. 1997, 2004), the development of a predictive model is unlikely in the foreseeable future.

The mechanism for introducing density dependence into the model has the unfortunate effect of introducing a low level of density dependence into the non-densitydependent transitions. This occurs when the transitions are standardized to sum to 1 . In some cases, there is obvious negative covariance between transition rates. For example, the $\mathrm{CC}$ transition is negatively dependent on free space, while the CI transition is positively dependent, and these two transitions come close to summing to a constant. This is the exception, however, and given the large number of transitions, it is difficult to find other pairs of transitions that covary. Hence it was decided to smear the negative covariance associated with a density-dependent transition across all other transitions from the same group.

Despite the highly complex nonlinear nature of the model, the only reason for multiple equilibrium points in the model community is the single anomalously high transition from free space to soft corals (Appendix A). If this transition is removed from the data set, then a simulation starting at $100 \%$ soft coral cover converges to the same equilibrium as all other simulations (results not shown), and the only difference to the results shown here is a slight decrease in soft coral cover at the expense of free space. This confirms the findings of our previous modeling studies (Tanner et al. 1994, 1996) that suggested that alternate stable states do not occur in this system. Indeed, even at the exposed crest, which experienced a major environmental change due to a cyclone, the community appears to be returning to something approximating its original state (Connell et al. 1997, 2004; J. E. Tanner, J. H. Connell, D. Greenberg, T. P. Hughes, A. M. Kerr, and C. C. Wallace, unpublished manuscript). However, if there is a persistent alteration to the environment, then an alternate (degraded) stable state may form (Connell 1997). For example, Hughes (1994) shows how persistent overfishing of grazers and the loss of herbivorous sea urchins has led to a phase shift from corals to algae in Jamaica. This contrasts with a study by Acevedo (1981) in which the final equilibrium reached was highly dependent on the starting composition for a densitydependent version of Horn's (1975) forest model. Similarly, Eppstein et al. (2006) found that community composition was sensitive to initial conditions when interactions occurred over a large spatial domain, but this sensitivity decreased as the spatial domain decreased.

The sensitivity analysis shows that while a large number of transitions are density dependent, very few have any noticeable effect on the community. A similar situation was found in a density-dependent population model of an intertidal zoanthid at a site nearby (Tanner 1999): density dependence was required in the transition from the largest to second largest size class, but was not needed in any other transition, to regulate population size to realistic levels between $80 \%$ and $90 \%$ cover. Wootton (2001) also showed that local density dependence had little influence on community composition for an intertidal mussel assemblage.

Total cover does not appear to be as good a predictor of transition probabilities as what the cover of individual species groups is. The percentage of variation explained in a given transition probability was almost always higher for individual species than for total cover (actually its converse was examined, free space), often by $20-30 \%$ or more, and for the few probabilities in which the reverse was true, total cover only explained 1-2\% more of the variation. Given some of the explanations provided here for density dependence in the transition probabilities, it is not surprising that total cover is insufficient to determine these probabilities. For instance, the size distribution of colonies within a species group, which is important as it determines competitive success, is less likely to be reflected in total cover than in that group's cover. The only studies to which this finding can be compared are those of forests in which density dependence is generally assumed to be determined by total stand basal area or some such measurement and not community composition (e.g., Buongiorno et al. 1995, Lin et al. 1996). It has been suggested on several occasions, however, that the intensity of competition in plant assemblages is determined by the total abundance of neighbors and not their identities (e.g., Zamfir and 
Goldberg 2000), in which case the use of stand basal area in forest models would be appropriate.

\section{Future directions}

While we have demonstrated the importance of density in determining the dynamics of these coral communities, its importance for individual coral populations has still to be determined. Although we have shown here that colonization of free space is generally determined by the cover of the colonizing species, it does not necessarily follow that the growth rates of individual colonies, or recruitment, will be density dependent. If colonies grow at a rate independent of density, an increase in the number of colonies will increase the amount of free space occupied by their growth. Similar situations apply to the other transitions in the model. Considering the importance of competition for space on coral reefs (reviewed in Lang and Chornesky [1990] and Karlson and Hurd [1993]), it is likely that population dynamics will be heavily influenced by total cover, if not population density. In high-diversity tropical forests, which are often considered to be the terrestrial equivalent of coral reefs, it appears that most species do exhibit conspecific density dependence in their population dynamics, despite the large number of other species present (Peters 2003). This finding contrasts with previous studies using the same data, as it employed a focal tree approach to determining surrounding density, rather than the traditional subplot approach (Peters 2003), which we also use here. Whether the plots used in the current study are large enough to take a focal colony approach is unclear. Feagin et al. (2005) and Eppstein et al. (2006) both found neighborhood size to be important in determining community dynamics of plant assemblages, and given the restricted distance over which corals are likely to compete, this approach will probably prove fruitful.

\section{ACKNOWLEDGMENTS}

We thank D. Greenberg, M. Spencer, and anonymous reviewers for comments on the manuscript. C. Wallace and B. Black generously assisted in the field over many years, along with many other volunteers. This research was funded by grants from the National Science Foundation (to J. H. Connell and T. P. Hughes) and the Australian Research Council (to T. P. Hughes).

\section{Literature Cited}

Abrams, P. A. 2001. Describing and quantifying interspecific interactions: a commentary on recent approaches. Oikos 94: 209-218.

Acevedo, M. F. 1981. On Horn's Markovian model of forest dynamics with particular reference to tropical forests. Theoretical Population Biology 19:230-250.

Aerts, L. A. M. 1998. Sponge/coral interactions in Caribbean reefs: analysis of overgrowth patterns in relation to species identity and cover. Marine Ecology Progress Series 175:241249.

Beisner, B. E., D. T. Haydon, and K. Cuddington. 2003. Alternative stable states in ecology. Frontiers in Ecology and the Environment 1:376-382.

Benedetti-Cecchi, L. 2000. Priority effects, taxonomic resolution, and the prediction of variable patterns of colonisation of algae in littoral rock pools. Oecologia 123:265-274.
Brace, R. C., and J. Pavey. 1978. Size-dependent dominance hierarchy in the anemone Actinia equina. Nature 273:752753.

Brook, B. W., and C. J. A. Bradshaw. 2006. Strength of evidence for density dependence in abundance time series of 1198 species. Ecology 87:1445-1451.

Buongiorno, J., J. Peyron, F. Houllier, and M. Bruciamacchie. 1995. Growth and management of mixed-species, unevenaged forests in the French Jura: implications for economic returns and tree diversity. Forestry Science 41:397-421.

Buss, L. W. 1980. Competitive intransitivity and size-frequency distributions of interacting populations. Proceedings of the National Academy of Sciences (USA) 77:5355-5359.

Caswell, H. 2001. Matrix population models. Second edition. Sinauer, Sunderland, Massachusetts, USA.

Caswell, H., and J. E. Cohen. 1995. Red, white and blue: environmental variance spectra and coexistence in metapopulations. Journal of Theoretical Biology 176:301-316.

Caswell, H., T. Takada, and C. M. Hunter. 2004. Sensitivity analysis of equilibrium in density-dependent matrix population models. Ecology Letters 7:380-387.

Connell, J. H. 1987. Change and persistence in some marine communities. Pages 339-352 in A. J. Gray, editor. Colonization, succession and stability. 26th Symposium of the British Ecolological Society. Blackwell Scientific, Oxford, UK.

Connell, J. H. 1997. Disturbance and recovery of coral assemblages. Coral Reefs 16:S101-S113.

Connell, J. H., T. P. Hughes, and C. C. Wallace. 1997. A $30-$ year study of coral abundance, recruitment, and disturbance at several scales in space and time. Ecological Monographs 67:489-507.

Connell, J. H., T. E. Hughes, C. C. Wallace, J. E. Tanner, K. E. Harms, and A. M. Kerr. 2004. A long-term study of competition and diversity of corals. Ecological Monographs 74:179-210.

Connell, J. H., and R. O. Slatyer. 1977. Mechanisms of succession in natural communities and their role in community stability and organisation. American Naturalist 111: 1119-1144.

Enright, N. J., and J. C. Ogden. 1979. Applications of transition matrix models in forest dynamics: Araucaria in Papua New Guinea and Nothofagus in New Zealand. Australian Journal of Ecology 4:3-23.

Eppstein, M. J., J. D. Bever, and J. Molofsky. 2006. Spatiotemporal community dynamics induced by frequency dependent interactions. Ecological Modelling 197:133-147.

Feagin, R. A., X. B. Wu, F. E. Smeins, S. G. Whisenant, and W. E. Grant. 2005. Individual versus community level processes and pattern formation in a model of sand dune plant succession. Ecological Modelling 183:435-449.

Goldberg, D. E., R. Turkington, L. Olsvig-Whittaker, and A. R. Dyer. 2001. Density dependence in an annual plant community: variation among life history stages. Ecological Monographs 71:423-446.

Grant, A., and T. G. Benton. 2003. Density-dependent populations require density-dependent elasticity analysis: an illustration using the LPA model of Tribolium. Journal of Animal Ecology 72:94-105.

Greene, C. H., and A. Schoener. 1982. Succession on marine hard substrata: a fixed lottery. Oecologia 55:289-297.

Harrison, P. L., and C. C. Wallace. 1990. Reproduction, dispersal and recruitment of scleractinian corals. Pages 133207 in Z. Dubinsky, editor. Ecosystems of the world. Elsevier, New York, New York, USA.

Hartshorn, G. S. 1978. Tree falls and tropical forest dynamics. Pages 617-638 in P. B. Tomlinson, editor. Tropical trees as living ecosystems. Cambridge University Press, Cambridge, UK.

Hastie, T. J., and R. J. Tibshirani. 1990. Generalised additive models. Chapman and Hall, London, UK. 
Hill, M. F., J. D. Witman, and H. Caswell. 2002. Spatiotemporal variation in Markov chain models of subtidal community succession. Ecology Letters 5:665-675.

Horn, H. S. 1975. Markovian properties of forest succession. Pages 196-211 in M. L. Cody, editor. Ecology and evolution of communities. Harvard University Press, Cambridge, Massachusetts, USA.

Hughes, T. P. 1994. Catastrophes, phase-shifts and large-scale degradation of a Carribean coral reef. Science 265:15471551 .

Karlson, R. H., and L. E. Hurd. 1993. Disturbance, coral reef communities, and changing ecological paradigms. Coral Reefs 12:117-126.

Lang, J. C., and E. A. Chornesky. 1990. Competition between scleractinian reef corals: a review of mechanisms and effects. Pages 209-252 in Z. Dubinsky, editor. Ecosystems of the world: coral reefs. Elsevier, Amsterdam, The Netherlands.

Lin, C. R., J. Buongiorno, and M. Vasievich. 1996. A multispecies, density-dependent matrix growth model to predict tree diversity and income in northern hardwood forests. Ecological Modelling 91:193-211.

Lough, T. J., J. B. Wilson, A. F. Mark, and A. C. Evans. 1987. Succession in a New Zealand alpine cushion community: a Markovian model. Vegetatio 71:129-138.

Manly, B. F. 1991. Randomization and Monte Carlo methods in biology. Chapman and Hall, London, UK.

Miller, T. E. X. 2007. Demographic models reveal the shape of density dependence for a specialist insect herbivore on variable host plants. Journal of Animal Ecology 76:722-729.

Molofsky, J., and J. D. Bever. 2002. A novel theory to explain species diversity in landscapes: positive frequency dependence and habitat suitability. Proceedings of the Royal Society 269: 2389-2393.

Pantastico-Caldas, M., and D. L. Venable. 1993. Competition in two species of desert annuals along a topographic gradient. Ecology 74:2192-2203.

Peters, H. A. 2003. Neighbour-regulated mortality: the influence of positive and negative density dependence on tree populations in species-rich tropical forests. Ecology Letters 6: 757-765.

Petraitis, P. S., and S. R. Dudgeon. 2004. Detection of alternative stable states in marine communities. Journal of Experimental Marine Biology and Ecology 300:343-371.
Prach, K., J. Leps, and J. Michálek. 1996. Establishment of Picea abies seedlings in a central European mountain grassland: an experimental study. Journal of Vegetation Science 7:681-684.

Spencer, M., and E. Susko. 2005. Continuous-time Markov models for species interactions. Ecology 86:3272-3278.

Spencer, M., and J. E. Tanner. 2008. Lotka-Volterra competition models for sessile organisms. Ecology 89:1134-1143.

Suding, K. N., and D. Goldberg. 2001. Do disturbances alter competitive hierarchies? Mechanisms of change following gap creation. Ecology 82:2133-2149.

Tanner, J. E. 1997. The effects of density on the zoanthid Palythoa caesia. Journal of Animal Ecology 66:793-810.

Tanner, J. E. 1999. Density dependent population dynamics in clonal organisms: a modelling approach. Journal of Animal Ecology 66:390-399.

Tanner, J. E., T. P. Hughes, and J. H. Connell. 1994. Species coexistence, keystone species and succession in coral assemblages: a sensitivity analysis. Ecology 75:2204-2219.

Tanner, J. E., T. P. Hughes, and J. H. Connell. 1996. The role of history in community dynamics: a modelling approach. Ecology 77:108-117.

Taylor, D. R., and L. W. Aarssen. 1989. On the density dependence of replacement-series competition experiments. Journal of Ecology 77:975-988.

Usher, M. B., and T. W. Parr. 1977. Are there successional changes in arthropod decomposer communities? Journal of Environmental Management 5:151-160.

Walker, L. R., and F. S. Chapin. 1987. Interactions among processes controlling successional change. Oikos 50:131-135.

Wang, G. M., N. T. Hobbs, R. B. Boone, A. W. Illius, I. J. Gordon, J. E. Gross, and K. L. Hamlin. 2006. Spatial and temporal variability modify density dependence in populations of large herbivores. Ecology 87:95-102.

Wootton, J. T. 2001. Local interactions predict large-scale pattern in empirically derived cellular automata. Nature 413: 841-844.

Wootton, J. T. 2004. Markov chain models predict the consequences of experimental extinctions. Ecology Letters $7: 653-660$.

Zamfir, M., and D. E. Goldberg. 2000. The effect of initial density on interactions between bryophytes at individual and community levels. Journal of Ecology 88:243-255.

\section{APPENDIX A}

Generalized additive model fits for transition probabilities at the protected crest (Ecological Archives E090-035-A1).

\section{APPENDIX B}

Sensitivity analysis for the protected crest (Ecological Archives E090-035-A2). 